\title{
Nutritional quality of foods and beverages on child-care centre menus in Mexico
}

\author{
Sara E Benjamin Neelon ${ }^{1, *}$, Hortensia Reyes-Morales ${ }^{2}$, Jess Haines ${ }^{3}$, \\ Matthew W Gillman ${ }^{4}$ and Elsie M Taveras ${ }^{4}$ \\ 'Department of Community and Family Medicine, Duke University Medical Center and Duke Global Health \\ Institute, 2200 W Main Street, DUMC 104006, Durham, NC 27705, USA: ${ }^{2}$ Center for Health Systems \\ Research, National Institute of Public Health, Cuernavaca, Mor Mexico, Mexico: ${ }^{3}$ Department of Family \\ Relations \& Applied Nutrition, University of Guelph, Guelph, Ontario, Canada: ${ }^{4}$ Obesity Prevention Program, \\ Department of Population Medicine, Harvard Medical School and Harvard Pilgrim Health Care Institute, \\ Boston, MA, USA
}

Submitted 14 January 2012: Final revision received 7 August 2012: Accepted 13 August 2012: First published online 4 0ctober 2012

\begin{abstract}
Objective: The purpose of the present study was to assess the nutritional quality of foods and beverages listed on menus serving children in government-sponsored child-care centres throughout Mexico.

Design: For this cross-sectional menu assessment, we compared (i) food groups and portion sizes of foods and beverages on the menus with MyPlate recommendations and (ii) macronutrients, sugar and fibre with Daily Reference Intake standards.

Setting: Menus reflected foods and beverages served to children attending one of 142 government-sponsored child-care centres throughout Mexico.

Subjects: There were fifty-four distinct menus for children aged 4-6 months, 7-9 months, 10-12 months, 13-23 months, 24-47 months and 48-72 months. Results: Menus included a variety of foods meeting minimum MyPlate recommendations for each food category except whole grains for children aged 48-72 months. Menus listed excessive amounts of high-energy beverages, including full-fat milk, fruit juice and sugar-sweetened beverages for children of all ages. The mean daily energy content of menu items yielded an average of $2.76 \mathrm{MJ}$ for infants, $4.77 \mathrm{MJ}$ for children aged 13-23 months, 5.36 MJ for children aged 24-47 months and $5 \cdot 87 \mathrm{MJ}$ for children aged 48-72 months. Foods and beverages on menus provided sufficient grams of carbohydrate and fat, but excessive protein.

Conclusions: Menus provided a variety of foods but excessive energy. Whole grains were limited, and high-energy beverages were prevalent. Both may be appropriate targets for nutrition intervention. Future studies should move beyond menus and assess what children actually consume in child care.
\end{abstract}

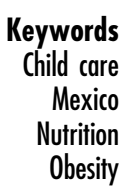

Mexico is in the midst of a nutrition transition ${ }^{(1,2)}$. Wasting and stunting among children $<5$ years of age have declined in recent years ${ }^{(3-7)}$, while rates of obesity have risen steadily ${ }^{(3,7-12)}$. Data from a recent national study in Mexico showed that $20 \%$ of children aged 2-12 years were overweight or obese ${ }^{(8)}$. For children of pre-school age, rates of obesity were highest in northern Mexico, in urban areas such as Mexico City and among children from higher-resource families ${ }^{(8,13,14)}$. Childhood obesity is associated with a number of adverse health outcomes that can include type 2 diabetes mellitus ${ }^{(15,16)}$, hypertension and hyperlipidaemia ${ }^{(15,17,18)}$, asthma and sleep apnoea ${ }^{(19-21)}$, early maturation ${ }^{(22,23)}$ and psychosocial stress ${ }^{(24-26)}$.

Dietary determinants of childhood obesity include excessive intake of sugar-sweetened beverages ${ }^{(27)}$, fruit juice $^{(28,29)}$ and high-energy foods of low nutritional value, such as chips and cookies ${ }^{(30-32)}$. A handful of previous studies have assessed dietary intake in young children in Mexico. In one study of public-school children in Mexico City, researchers found that children consumed insufficient fibre $(<25 \mathrm{~g} / \mathrm{d})$ and excessive dietary fat $(>35 \%$ of daily energy) ${ }^{(18)}$. A second study of children 10-19 years of age in Mexico City found that foods provided by schools were of lower nutritional quality than those brought from home ${ }^{(33)}$. A study of children aged 1-4 years showed that those living in Mexico City consumed more dietary fat, carbohydrate and total energy than children in any other region in Mexico ${ }^{(34)}$. In a national study of beverage consumption, researchers noted a substantial increase in caloric beverage intake over the past decade 
among pre-school and school-aged children ${ }^{(35)}$. The authors cited full-fat milk and sugar-sweetened fruit juice as the two primary beverages consumed by young children. However, little is known about dietary intake and the nutritional quality of foods and beverages provided to children outside the family home. The purpose of the present study was to assess the nutritional quality of foods and beverages listed on menus serving children in governmentsponsored child-care centres throughout Mexico.

\section{Methods}

We reviewed and analysed a sample of fifty-four daily menus (including breakfast, lunch and afternoon snack) reflecting foods and beverages served to children attending 142 government-sponsored child-care centres in Mexico, all of whom follow the same menus. The 142 child-care centres provide care to almost 30000 children from 6 weeks to 6 years of age. We collected menus in the spring of 2009. Menus were still in use in early 2012, but are scheduled to be re-evaluated later that year. Menus were developed by a nutritionist and were used by all government-sponsored child-care centres throughout the country. Menus varied by age, with separate menus for children aged 4-6 months, 7-9 months, 10-12 months, 13-23 months, 24-47 months and 48-72 months. A $1 \mathrm{~d}$ sample menu is provided in Fig. 1. We translated menus from Spanish to English prior to nutrient analysis. Since this research was limited to a menu review and did not involve human subjects, we were granted an exemption from the Institutional Review Boards at both Harvard Pilgrim Health Care and Duke University Medical Center.

To assess the nutritional value of foods and beverages listed on the menus, we analysed menus using the Nutrition Data System for Research (NDS-R) software version 2005 (Nutrition Coordinating Center, University of Minnesota, Minneapolis, MN, USA). The NDS-R software generated serving sizes based on the 1992 US Department of Agriculture (USDA) Food Guide Pyramid, a national nutrition guideline system in the USA. Menus specified amounts of foods (e.g. $50 \mathrm{~g}$ beans) and beverages (e.g. $120 \mathrm{ml}$ fruit juice) for children in each age group. The menus also included detailed ingredients for mixed dishes, which we used in the nutrient analysis. We translated serving sizes into cups and ounces for comparison with national recommendations. Generally, information about the amounts and types of foods was included on the menus. In some cases, the method of preparation, the recipe and the list of ingredients were also included. A small number of traditional Mexican foods were listed on the menus but not included in the NDS-R database. In those cases, we used the closest available substitute based on expert review by one author (e.g. Garibaldi biscuit was substituted with vanilla sponge cake). The NDS-R software does not categorize food into various groups (e.g. fruits); we therefore manually combined data into food group categories. Because NDS-R classified sweetened beverages (e.g. fruit drinks, flavoured waters, sweetened milks) and $100 \%$ fruit juices by their ingredient components, we created a separate category for servings of juice and sugarsweetened beverages.

We reviewed menu data to determine the amount and type of foods and beverages served to children by age group, averaged over one full day of care. These menus, however, do not reflect foods and beverages served to children at home or outside child care. First, we compared food group values with current USDA MyPlate ${ }^{(36)}$ recommendations by age group. We used MyPlate recommendations because, at the time of the study, Mexico did not have national food group standards for children, with the exception of La Piramide De La Salud, which did not include specific recommendations to allow for comparisons. MyPlate recommendations represent the 2010 Dietary Guidelines for Americans food group serving sizes that replaced the USDA Food Guide Pyramid in 2011. We compared portion sizes of foods and beverages on the menus with daily MyPlate recommendations for each food group (grains, vegetables, fruits, meats and dairy). Next, we computed mean total energy, macronutrients (fat, carbohydrate and protein), fibre and sugar for each menu by age group, and compared these values with the Daily Reference Intake (DRI) ${ }^{(37)}$ standards. Since MyPlate and DRI recommendations begin at age 2 years, with a few exceptions noted, we were not able to make many comparisons for dietary data from infant menus.

\section{Results}

Based on a total of fifty-four menus among children aged 24-72 months, for whom MyPlate food group recommendations exist, children in child-care centres were provided with sufficient servings of grains, vegetables, fruits, meats and meat alternatives, and dairy (Table 1). Foods listed contained some wholegrain items, but amounts were below recommendations for children aged 48-72 months. Children received more than the recommended amounts of dark (orange, red, yellow) vegetables, and these vegetables represented $\sim 50 \%$ of the total vegetables listed on the menus. Moreover, fried vegetables such as French fries and fried potatoes were not listed on any of the $54 \mathrm{~d}$ of menus. Nearly half of the meats and meat alternatives on the menus were high-fat or fried, with high-fat beef and eggs being the most common meat items. Fewer than $4 \%$ of total dairy items were reduced-fat and none were $1 \%$ or fat-free. Highsugar and high-salt snack foods were not often listed on the menus.

In terms of beverages, menus included some fruit juice for all age groups. Infants were provided a mean of 
MENÚ DESARROLLADO 14

Ración de alimentos a servi para niños de 10 a 23 meses

\begin{tabular}{|c|c|c|c|c|c|}
\hline DESAYUNO & RACIONES 1 & COMIDA & RACIONES 1 & MERIENDA & RACIONES 1 \\
\hline \multirow{2}{*}{ Fruit De Temporada } & $150 \mathrm{~g}$ & Sopa De Codito & & Fruta De Temporado & $150 \mathrm{~g}$ \\
\hline & & Pasta de codito & $15 \mathrm{~g}$ & & \\
\hline Molletes Con Salsa Mexicana & & Margarina sin sal & $3 \mathrm{~g}$ & Pollo a La Mantequilla & \\
\hline Pan blanco bolillo & $35 \mathrm{~g}$ & Apio & $5 \mathrm{~g}$ & Pechuga de pollo & $60 \mathrm{~g}$ \\
\hline Frijol & $15 \mathrm{~g}$ & Calabacita & $15 \mathrm{~g}$ & Ajo & $0.0002 \mathrm{~g}$ \\
\hline Queso manchego & $30 \mathrm{~g}$ & Papa & $10 \mathrm{~g}$ & Cebolla & $1 \mathrm{~g}$ \\
\hline Cebolla & $2 \mathrm{~g}$ & Tocino & $3 \mathrm{~g}$ & Margarina sin sal & $5 \mathrm{~g}$ \\
\hline Sal de mesa & $1 \mathrm{~g}$ & Jitomate & $30 \mathrm{~g}$ & Sal de mesa & $1 \mathrm{~g}$ \\
\hline Jitomate & $30 \mathrm{~g}$ & Consumé de pollo & $0.0003 \mathrm{~g}$ & & \\
\hline Aceite de cártamo & $5 \mathrm{ml}$ & Aceite de cártamo & $1 \mathrm{ml}$ & Ensalada De Betabel & \\
\hline \multirow[t]{2}{*}{ Margarina sin sal } & $1 \mathrm{~g}$ & Sal de mesa & $1 \mathrm{~g}$ & Betabel & $60 \mathrm{~g}$ \\
\hline & & Cebolla & $1 \mathrm{~g}$ & Naranja & $50 \mathrm{~g}$ \\
\hline Leche Con Cajeta & & Ajo & $0.0001 \mathrm{~g}$ & Azúcar & $10 \mathrm{~g}$ \\
\hline Leche entera polvo & $18 \mathrm{~g}$ & Hierbas de olor tomillo & $0.0001 \mathrm{~g}$ & & \\
\hline Cajeta & $15 \mathrm{~g}$ & & & Leche & \\
\hline \multirow[t]{2}{*}{ Agua } & $150 \mathrm{ml}$ & Loma a La Naranja & & Leche entera polvo & $18 \mathrm{~g}$ \\
\hline & & Lomo de cerdo & $80 \mathrm{~g}$ & Agua & $150 \mathrm{ml}$ \\
\hline Jugo De Piña* & & Naranja para jugo & $50 \mathrm{~g}$ & Azúcar & $10 \mathrm{~g}$ \\
\hline Concentrado de piña & $15 \mathrm{ml}$ & Perejil & $2 \mathrm{~g}$ & & \\
\hline \multirow[t]{25}{*}{ Agua } & $60 \mathrm{ml}$ & Pimienta negra polvo & $0.0001 \mathrm{~g}$ & Tortilla De Harina & $25 \mathrm{~g}$ \\
\hline & & Hierbas de olor & $0.0001 \mathrm{~g}$ & & \\
\hline & & Naranja & $20 \mathrm{~g}$ & Agua De Jamaica & \\
\hline & & Cebolla & $1 \mathrm{~g}$ & Flor De Jamaica & $3 \mathrm{~g}$ \\
\hline & & Sal de mesa & $1 \mathrm{~g}$ & Azúcar & $10 \mathrm{~g}$ \\
\hline & & Aceite de cártamo & $3 \mathrm{ml}$ & Agua & $150 \mathrm{ml}$ \\
\hline & & Ensalada Tricolor & & Jugo De Piña* & \\
\hline & & Zanahoria & $20 \mathrm{~g}$ & Concentrado de piña & $15 \mathrm{ml}$ \\
\hline & & Pepino & $35 \mathrm{~g}$ & Agua & $60 \mathrm{ml}$ \\
\hline & & Pimiento morrón rojo & $10 \mathrm{~g}$ & & \\
\hline & & Limón & $5 \mathrm{~g}$ & & \\
\hline & & Sal de mesa & $1 \mathrm{~g}$ & & \\
\hline & & & & & \\
\hline & & Malvavisco Cereal & & & \\
\hline & & Cereal & $30 \mathrm{~g}$ & & \\
\hline & & Malvavisco & $15 \mathrm{~g}$ & & \\
\hline & & Margarina sin sal & $8 \mathrm{~g}$ & & \\
\hline & & Extracto de vainilla & $2 \mathrm{ml}$ & & \\
\hline & & & & & \\
\hline & & Pan De Caja Integral & $25 \mathrm{~g}$ & & \\
\hline & & & & & \\
\hline & & Agua De Mandarina & & & \\
\hline & & $\begin{array}{l}\text { Concentrado de } \\
\text { mandarina }\end{array}$ & $10 \mathrm{ml}$ & & \\
\hline & & Azúcar & $10 \mathrm{~g}$ & & \\
\hline & & Aqua & $150 \mathrm{ml}$ & & \\
\hline
\end{tabular}

${ }^{*}$ Calcular sólo para niños de 10 a 12 meses.

Fig. 1 Sample of a $1 \mathrm{~d}$ menu for children aged 10-23 months attending child-care centres in Mexico, spring 2009

$1 \cdot 6$ (sD 0.2) oz (4-6 months), $4 \cdot 9$ (sD 0.3) oz (7-9 months) and $1 \cdot 0(\mathrm{SD} 0 \cdot 0) \mathrm{Oz}(10-12$ months) of $100 \%$ juice daily. Older children received $<1 \mathrm{oz} / \mathrm{d}$, on average. Other sugar-sweetened beverages, such as water or milk with added sugar, were more common, with menus listing $6.9(\mathrm{SD} 0 \cdot 0) \mathrm{oz} / \mathrm{d}$ for infants aged 10-12 months and $13 \cdot 3(\mathrm{sD} 0 \cdot 1) \mathrm{oz} / \mathrm{d}$ for children aged 13-72 months of age. Full-fat milk, including both sweetened and unsweetened, was the most common type of milk served to children of all ages. For children 13-72 months of age, menus listed an average of $1 \cdot 2$ cups of full-fat milk/d, with small amounts of reduced-fat milk. Of all milk served as a beverage (not in recipes), 37\% had added sugars. Children $<1$ year of age were also served whole cow's milk.

Menus yielded a mean daily energy content of $2 \cdot 10$ (sD 0 09) MJ for infants aged 4-6 months, 3.03 (sD 0 13) MJ for infants aged 7-9 months and 3.14 (SD 0 48) MJ for infants aged 10-12 months. For older children, menus provided $4 \cdot 77$ (SD 0.68) MJ/d for children aged 13-23 months, $5.36(\mathrm{sD} 0.73) \mathrm{MJ} / \mathrm{d}$ for children aged 24-47 months and $5 \cdot 87(\mathrm{sD} 0 \cdot 70) \mathrm{MJ} / \mathrm{d}$ for children aged 48-72 months (Table 2). Menus provided a mean daily fat amount of $20 \cdot 7$ (SD 1.2) g for infants aged 4-6 months, $28 \cdot 6$ (sD 1.6) g for infants aged 7-9 months and $33 \cdot 3$ (SD 7·8) $\mathrm{g}$ for infants 
Table 1 Foods and beverages from fifty-four Mexican child-care centre menus compared with MyPlate recommendations by age*

\begin{tabular}{|c|c|c|c|c|c|}
\hline \multirow[b]{2}{*}{ Food category } & \multirow[b]{2}{*}{ Age (months) } & \multicolumn{2}{|c|}{ Amount provided } & \multirow[b]{2}{*}{ MyPlate recommendation } & \multirow[b]{2}{*}{ Percentage of MyPlate } \\
\hline & & Mean & SD & & \\
\hline \multirow[t]{6}{*}{ Grains, total (oz) } & $4-6$ & 0.2 & $0 \cdot 1$ & \multicolumn{2}{|c|}{ No MyPlate recommendation } \\
\hline & $7-9$ & $1 \cdot 4$ & 0.3 & & \\
\hline & $10-12$ & $1 \cdot 7$ & 0.8 & & \\
\hline & $13-23$ & $2 \cdot 4$ & $0 \cdot 7$ & & \\
\hline & $24-47$ & $2 \cdot 9$ & 0.9 & $4 \mathrm{oz}$ & 73 \\
\hline & $48-72$ & $3 \cdot 2$ & 0.9 & $5 \mathrm{oz}$ & 64 \\
\hline \multirow[t]{6}{*}{ All or some whole grains (oz) } & $4-6$ & 0.0 & 0.0 & \multicolumn{2}{|c|}{ No MyPlate recommendation } \\
\hline & $7-9$ & $0 \cdot 7$ & 0.5 & & \\
\hline & $10-12$ & 0.5 & 0.5 & & \\
\hline & $13-23$ & $0 \cdot 8$ & 0.5 & & \\
\hline & $24-47$ & $1 \cdot 1$ & $0 \cdot 8$ & $2 \mathrm{oz}$ & 55 \\
\hline & $48-72$ & $1 \cdot 2$ & $0 \cdot 8$ & $2.5 \mathrm{oz}$ & 48 \\
\hline Vegetables, total (cups) & $4-6$ & $0 \cdot 4$ & 0.2 & No MyPlate rec & mmendation \\
\hline & $7-9$ & 0.8 & $0 \cdot 3$ & & \\
\hline & $10-12$ & $0 \cdot 8$ & 0.4 & & \\
\hline & $13-23$ & 0.9 & 0.4 & & \\
\hline & $24-47$ & $1 \cdot 0$ & 0.5 & 1.5 cups & 75 \\
\hline & $48-72$ & $1 \cdot 1$ & 0.5 & 1.5 cups & 73 \\
\hline Vegetables, dark (cups)† & $4-6$ & $0 \cdot 1$ & $0 \cdot 1$ & No MyPlate rec & mmendation \\
\hline & $7-9$ & 0.3 & 0.3 & & \\
\hline & $10-12$ & 0.4 & 0.3 & & \\
\hline & $13-23$ & 0.5 & 0.4 & & \\
\hline & $24-47$ & 0.6 & 0.4 & 0.5 cups & 120 \\
\hline & $48-72$ & 0.6 & 0.4 & 0.5 cups & 120 \\
\hline Vegetables, starchy (not fried) (cups) & $4-6$ & $0 \cdot 1$ & 0.2 & No MyPlate rec & mmendation \\
\hline & $7-9$ & $0 \cdot 1$ & $0 . \overline{2}$ & & \\
\hline & $10-12$ & $0 \cdot 1$ & $0 \cdot 1$ & & \\
\hline & $13-23$ & $0 \cdot 2$ & $0 \cdot 1$ & & \\
\hline & $24-47$ & $0 \cdot 2$ & 0.2 & 0.3 cups & 67 \\
\hline & $48-72$ & 0.2 & 0.2 & $0 \cdot 3$ cups & 67 \\
\hline Vegetables, other (cups) & $4-6$ & $0 \cdot 2$ & 0.3 & No MyPlate rec & mmendation \\
\hline & $7-9$ & $0 \cdot 4$ & 0.3 & & \\
\hline & $10-12$ & $0 \cdot 2$ & 0.2 & & \\
\hline & $13-23$ & 0.3 & 0.2 & & \\
\hline & $24-47$ & $0 \cdot 3$ & 0.2 & 0.7 cups & 67 \\
\hline & $48-72$ & 0.3 & 0.2 & $0 \cdot 7$ cups & 43 \\
\hline Vegetables, fried (cups) & $4-6$ & 0.0 & 0.0 & No MyPlate rec & mmendation \\
\hline & 7-9 & $0 \cdot 0$ & 0.0 & & \\
\hline & $10-12$ & $0 \cdot 0$ & 0.0 & & \\
\hline & $13-23$ & $0 \cdot 0$ & 0.0 & & \\
\hline & $24-47$ & $0 \cdot 0$ & 0.0 & & \\
\hline & $48-72$ & $0 \cdot 0$ & 0.0 & & \\
\hline Fruit (excluding $100 \%$ juice) (cups) & $4-6$ & 0.6 & $0 \cdot 1$ & No MyPlate rec & mmendation \\
\hline & $7-9$ & $0 \cdot 8$ & 0.2 & & \\
\hline & $10-12$ & $0 \cdot 7$ & 0.2 & & \\
\hline & $13-23$ & $0 \cdot 7$ & 0.2 & & \\
\hline & $24-47$ & $0 \cdot 9$ & 0.3 & $1 \cdot 0$ cups & 90 \\
\hline & $48-72$ & $1 \cdot 0$ & 0.3 & 1.5 cups & 67 \\
\hline Meats/alternatives, total (oz) & $4-6$ & 0.0 & 0.0 & No MyPlate rec & mmendation \\
\hline & $7-9$ & $1 \cdot 3$ & $0 \cdot 1$ & & \\
\hline & $10-12$ & $2 \cdot 8$ & 0.8 & & \\
\hline & $13-23$ & 3.5 & $1 \cdot 2$ & & \\
\hline & $24-47$ & $4 \cdot 0$ & $1 \cdot 2$ & $3 \mathrm{oz}$ & 72 \\
\hline & $48-72$ & $4 \cdot 4$ & $1 \cdot 3$ & $4 \mathrm{oz}$ & 110 \\
\hline Meat, high-fat/fried (oz) & $4-6$ & 0.0 & 0.0 & No MyPlate rec & mmendation \\
\hline & $7-9$ & $0 \cdot 0$ & $0 \cdot 1$ & & \\
\hline & $10-12$ & $1 \cdot 3$ & $0 \cdot 8$ & & \\
\hline & $13-23$ & $1 \cdot 7$ & $1 \cdot 2$ & & \\
\hline & $24-47$ & $1 \cdot 9$ & $1 \cdot 2$ & & \\
\hline & $48-72$ & $2 \cdot 0$ & $1 \cdot 4$ & & \\
\hline Meat, low-fat (oz) & $4-6$ & 0.0 & 0.0 & No MyPlate rec & mmendation \\
\hline & $7-9$ & $0 \cdot 7$ & 0.0 & & \\
\hline & $10-12$ & $0 \cdot 7$ & 0.7 & & \\
\hline & $13-23$ & 0.8 & 0.8 & & \\
\hline & $24-47$ & 0.9 & $1 \cdot 0$ & & \\
\hline & $48-72$ & $1 \cdot 1$ & $1 \cdot 1$ & & \\
\hline Meat alternatives (oz) & $4-6$ & 0.0 & 0.0 & No MyPlate rec & mmendation \\
\hline & $7-9$ & 0.6 & 0.0 & & \\
\hline & $10-12$ & 0.9 & 0.9 & & \\
\hline & $13-23$ & $1 \cdot 0$ & $1 \cdot 1$ & & \\
\hline & $24-47$ & $1 \cdot 2$ & 0.6 & & \\
\hline & $48-72$ & $1 \cdot 3$ & $0 \cdot 7$ & & \\
\hline
\end{tabular}


Table 1 Continued

\begin{tabular}{|c|c|c|c|c|c|}
\hline \multirow[b]{2}{*}{ Food category } & \multirow[b]{2}{*}{ Age (months) } & \multicolumn{2}{|c|}{ Amount provided } & \multirow[b]{2}{*}{ MyPlate recommendation } & \multirow[b]{2}{*}{ Percentage of MyPlate } \\
\hline & & Mean & SD & & \\
\hline \multirow[t]{6}{*}{ Dairy, total (excluding infant formula) (cups)‡ } & $4-6$ & $0 \cdot 1$ & $0 \cdot 1$ & \multicolumn{2}{|c|}{ No MyPlate recommendation } \\
\hline & $7-9$ & $0 \cdot 3$ & $0 \cdot 1$ & & \\
\hline & $10-12$ & 0.5 & $0 \cdot 4$ & & \\
\hline & $13-23$ & $1 \cdot 7$ & 0.5 & & \\
\hline & $24-47$ & $1 \cdot 8$ & $0 \cdot 6$ & 2.5 cups & 72 \\
\hline & $48-72$ & 1.9 & $0 \cdot 7$ & \multirow{7}{*}{\multicolumn{2}{|c|}{ No MyPlate recommendation }} \\
\hline \multirow[t]{6}{*}{ Milk, whole (cups) } & $4-6$ & $0 \cdot 1$ & $0 \cdot 1$ & & \\
\hline & $7-9$ & $0 \cdot 3$ & $0 \cdot 1$ & & \\
\hline & $10-12$ & $1 \cdot 0$ & 0.2 & & \\
\hline & $13-23$ & $1 \cdot 2$ & $0 \cdot 2$ & & \\
\hline & $24-47$ & $1 \cdot 2$ & 0.2 & & \\
\hline & $48-72$ & $1 \cdot 2$ & $0 \cdot 2$ & & \\
\hline \multirow[t]{6}{*}{ Milk, reduced-fat (cups) } & $4-6$ & $0 \cdot 0$ & 0.0 & \multirow{6}{*}{\multicolumn{2}{|c|}{ No MyPlate recommendation }} \\
\hline & $7-9$ & $0 \cdot 0$ & 0.0 & & \\
\hline & $10-12$ & $0 \cdot 1$ & $0 \cdot 1$ & & \\
\hline & $13-23$ & $0 \cdot 1$ & $0 \cdot 1$ & & \\
\hline & $24-47$ & $0 \cdot 1$ & $0 \cdot 1$ & & \\
\hline & $48-72$ & $0 \cdot 1$ & $0 \cdot 1$ & & \\
\hline \multirow[t]{6}{*}{ Milk, $1 \%$ or non-fat (cups) } & $4-6$ & $0 \cdot 0$ & $0 \cdot 0$ & \multirow{6}{*}{\multicolumn{2}{|c|}{ No MyPlate recommendation }} \\
\hline & $7-9$ & 0.0 & 0.0 & & \\
\hline & $10-12$ & $0 \cdot 0$ & $0 \cdot 0$ & & \\
\hline & $13-23$ & $0 \cdot 0$ & $0 \cdot 0$ & & \\
\hline & $24-47$ & $0 \cdot 0$ & 0.0 & & \\
\hline & $48-72$ & $0 \cdot 0$ & $0 \cdot 0$ & & \\
\hline \multirow[t]{4}{*}{ Infant formula (oz) } & $4-6$ & $17 \cdot 7$ & $1 \cdot 2$ & \multirow{4}{*}{\multicolumn{2}{|c|}{ No MyPlate recommendation }} \\
\hline & $7-9$ & $12 \cdot 2$ & 0.0 & & \\
\hline & $10-12$ & 0.0 & $0 \cdot 0$ & & \\
\hline & $13-23$ & 0.0 & $0 \cdot 0$ & & \\
\hline \multirow[t]{6}{*}{$100 \%$ fruit juice $(o z) \S$} & $4-6$ & $1 \cdot 6$ & 0.2 & $0 \mathrm{oz}$ & \\
\hline & $7-9$ & 4.9 & 0.3 & $\leq 6 \mathrm{oz}$ & \\
\hline & $10-12$ & $1 \cdot 0$ & 0.0 & $\leq 6 \mathrm{oz}$ & 16 \\
\hline & $13-23$ & $0 \cdot 6$ & $0 \cdot 0$ & $\leq 6 \mathrm{oz}$ & 11 \\
\hline & $24-47$ & 0.6 & $0 \cdot 0$ & $\leq 6 \mathrm{oz}$ & 11 \\
\hline & $48-72$ & 0.6 & 0.0 & $\leq 6 \mathrm{oz}$ & 11 \\
\hline Sugar-sweetened beverages (oz)‡ & $4-6$ & $0 \cdot 0$ & $0 \cdot 0$ & No MyPlate rec & mmendation \\
\hline & $7-9$ & $0 \cdot 0$ & 0.0 & & \\
\hline & $10-12$ & $6 \cdot 9$ & $0 \cdot 0$ & & \\
\hline & $13-23$ & $13 \cdot 3$ & $0 \cdot 1$ & & \\
\hline & $24-47$ & $13 \cdot 3$ & $0 \cdot 1$ & & \\
\hline & $48-72$ & $13 \cdot 3$ & $0 \cdot 1$ & & \\
\hline Sugar snacks (FDA serving size) & $4-6$ & $0 \cdot 1$ & $0 \cdot 2$ & No MyPlate rec & mmendation \\
\hline & $7-9$ & $0 \cdot 0$ & 0.0 & & \\
\hline & $10-12$ & 0.2 & 0.3 & & \\
\hline & $13-23$ & $0 \cdot 2$ & $0 \cdot 4$ & & \\
\hline & $24-47$ & $0 \cdot \overline{3}$ & 0.5 & & \\
\hline & $48-72$ & $0 \cdot 3$ & $0 \cdot 6$ & & \\
\hline Salty snacks (FDA serving size) & $4-6$ & $0 \cdot 0$ & $0 \cdot 0$ & No MyPlate rec & mmendation \\
\hline & $7-9$ & $0 \cdot 0$ & $0 \cdot 0$ & & \\
\hline & $10-12$ & $0 \cdot 0$ & 0.0 & & \\
\hline & $13-23$ & $0 \cdot 0$ & $0 \cdot 0$ & & \\
\hline & $24-47$ & $0 \cdot 0$ & $0 \cdot 0$ & & \\
\hline & $48-72$ & $0 \cdot 0$ & $0 \cdot 0$ & & \\
\hline Added fats (FDA serving size) & $4-6$ & $0 \cdot 0$ & $0 \cdot 0$ & No MyPlate rec & mmendation \\
\hline & $7-9$ & 0.7 & 0.2 & & \\
\hline & $10-12$ & $3 \cdot 0$ & $1 \cdot 0$ & & \\
\hline & $13-23$ & $3 \cdot 6$ & $1 \cdot 2$ & & \\
\hline & $24-48$ & $4 \cdot 1$ & $1 \cdot 3$ & & \\
\hline & $45-72$ & $4 \cdot 4$ & $1 \cdot 4$ & & \\
\hline
\end{tabular}

FDA, Food and Drug Administration.

*MyPlate recommendations start at age 24 months. Age $24-47$ months is based on a $5.02 \mathrm{MJ}(1200 \mathrm{kcal})$ diet as recommended by MyPlate (2010 Dietary Guidelines for Americans) for a 3-year-old girl, active 30-60 min/d. Age 48-72 months is based on a 5.86 MJ (1400 kcal) diet as recommended by MyPlate (2010 Dietary Guidelines for Americans) for a 5-year-old girl, active 30-60 min/d.

tBased on amounts recommended weekly by MyPlate (2010 Dietary Guidelines for Americans). Dark vegetable recommendation combines green vegetables and orange vegetables.

$\neq$ Sweetened milk is categorized under both the dairy and sweetened beverage categories. Of sweetened beverages, sweetened milk accounts for $27 \%$ (3.6 oz) of the total for age 13-72 months and $0 \%$ for age 10-12 months.

$\$ 100 \%$ fruit juice served as a beverage only, not as added in a recipe. 
Table 2 Nutrients from fifty-four Mexican child-care centre menus compared with Dietary Reference Intake (DRI) standards by age*

\begin{tabular}{|c|c|c|c|c|c|}
\hline \multirow[b]{2}{*}{ Nutrient } & \multirow[b]{2}{*}{ Age (months) } & \multicolumn{2}{|c|}{ Amount provided } & \multirow[b]{2}{*}{ DRI standard } & \multirow[b]{2}{*}{ Percentage of DRI } \\
\hline & & Mean & SD & & \\
\hline \multirow[t]{6}{*}{ Energy $(\mathrm{MJ})^{\star}$} & $4-6$ & $2 \cdot 1$ & $0 \cdot 1$ & \multicolumn{2}{|l|}{ No DRI standard } \\
\hline & $7-9$ & $3 \cdot 0$ & $0 \cdot 1$ & & \\
\hline & $10-12$ & $3 \cdot 1$ & 0.5 & & \\
\hline & $13-23$ & $4 \cdot 8$ & $0 \cdot 7$ & $4 \cdot 18-5 \cdot 86 \mathrm{MJ}$ & $81-114$ \\
\hline & $24-47$ & $5 \cdot 4$ & $0 \cdot 7$ & $4 \cdot 18-5 \cdot 86 \mathrm{MJ}$ & $91-128$ \\
\hline & $48-72$ & $5 \cdot 9$ & $0 \cdot 7$ & $5 \cdot 86-6 \cdot 69 \mathrm{MJ}$ & $88-100$ \\
\hline \multirow{6}{*}{ Fat $(g)^{*}$} & $4-6$ & $20 \cdot 7$ & $1 \cdot 2$ & $31 \mathrm{~g}$ & 67 \\
\hline & $7-9$ & $28 \cdot 6$ & $1 \cdot 6$ & $30 \mathrm{~g}$ & 95 \\
\hline & $10-12$ & $33 \cdot 3$ & $7 \cdot 8$ & $30 \mathrm{~g}$ & 111 \\
\hline & $13-23$ & $50 \cdot 1$ & $9 \cdot 4$ & \multicolumn{2}{|l|}{ No DRI standard } \\
\hline & $24-47$ & $55 \cdot 8$ & $10 \cdot 7$ & & \\
\hline & $48-72$ & $60 \cdot 5$ & $10 \cdot 8$ & & \\
\hline \multirow[t]{6}{*}{ Percentage of energy from fatt } & $4-6$ & $37 \cdot 3$ & - & \multicolumn{2}{|l|}{ No DRI standard } \\
\hline & $7-9$ & $35 \cdot 5$ & - & & \\
\hline & $10-12$ & $39 \cdot 6$ & - & & \\
\hline & $13-23$ & $39 \cdot 5$ & - & $30-40 \% \ddagger$ & - \\
\hline & $24-47$ & $39 \cdot 0$ & - & $30-40 \% \ddagger$ & - \\
\hline & $48-72$ & $38 \cdot 7$ & - & $25-35 \% \ddagger$ & - \\
\hline \multirow[t]{6}{*}{ Saturated fat $(\mathrm{g})$} & $4-6$ & $7 \cdot 2$ & $0 \cdot 4$ & No DRI standard & \\
\hline & $7-9$ & $9 \cdot 2$ & $0 \cdot 3$ & & \\
\hline & $10-12$ & $9 \cdot 1$ & $3 \cdot 1$ & & \\
\hline & $13-23$ & $16 \cdot 2$ & $3 \cdot 4$ & & \\
\hline & $24-47$ & $17 \cdot 9$ & $4 \cdot 3$ & & \\
\hline & $48-72$ & $19 \cdot 2$ & $4 \cdot 4$ & & \\
\hline Percentage of energy from saturated fat & $4-6$ & $12 \cdot 9$ & - & No DRI standard & \\
\hline & $7-9$ & $11 \cdot 5$ & - & & \\
\hline & $10-12$ & $10 \cdot 8$ & - & & \\
\hline & $13-23$ & $12 \cdot 8$ & - & & \\
\hline & $24-47$ & $12 \cdot 6$ & - & & \\
\hline & $48-72$ & $12 \cdot 3$ & - & & \\
\hline Carbohydrate $(\mathrm{g})^{\star}$ & 4-6 & $70 \cdot 3$ & 5.9 & $60 \mathrm{~g}$ & 171 \\
\hline & 7-9 & $100 \cdot 4$ & $8 \cdot 1$ & $95 \mathrm{~g}$ & 106 \\
\hline & $10-12$ & $84 \cdot 1$ & $14 \cdot 0$ & $95 \mathrm{~g}$ & 89 \\
\hline & $13-23$ & $128 \cdot 0$ & $23 \cdot 1$ & $130 \mathrm{~g}$ & 99 \\
\hline & $24-47$ & $145 \cdot 0$ & $23 \cdot 4$ & $130 \mathrm{~g}$ & 116 \\
\hline & $48-72$ & $160 \cdot 4$ & $25 \cdot 4$ & $130 \mathrm{~g}$ & 123 \\
\hline Percentage of energy from carbohydrate & $4-6$ & $56 \cdot 1$ & - & No DRI standard & \\
\hline & 7-9 & $55 \cdot 3$ & - & & \\
\hline & $10-12$ & $45 \cdot 1$ & - & & \\
\hline & $13-23$ & $45 \cdot 0$ & - & $45-65 \% \ddagger$ & - \\
\hline & $24-47$ & $45 \cdot 4$ & - & $45-65 \% \neq$ & - \\
\hline & $48-72$ & $45 \cdot 8$ & - & $45-65 \% \ddagger$ & - \\
\hline Protein $(\mathrm{g})^{\star}$ & $4-6$ & $9 \cdot 9$ & 0.5 & $9 \cdot 1 \mathrm{~g}$ & 109 \\
\hline & 7-9 & $19 \cdot 8$ & $0 \cdot 4$ & $11 \mathrm{~g}$ & 180 \\
\hline & $10-12$ & $32 \cdot 3$ & $7 \cdot 0$ & $11 \mathrm{~g}$ & 294 \\
\hline & $13-23$ & $48 \cdot 8$ & $8 \cdot 8$ & $13 \mathrm{~g}$ & 375 \\
\hline & $24-47$ & $54 \cdot 8$ & $9 \cdot 9$ & $13 \mathrm{~g}$ & 422 \\
\hline & $48-72$ & $59 \cdot 9$ & $10 \cdot 0$ & $19 \mathrm{~g}$ & 315 \\
\hline Percentage of energy from proteint & $4-6$ & $7 \cdot 9$ & - & No DRI standard & \\
\hline & $7-9$ & $10 \cdot 9$ & - & & \\
\hline & $10-12$ & $17 \cdot 2$ & - & & \\
\hline & $13-23$ & $17 \cdot 2$ & - & $5-20 \% \ddagger$ & - \\
\hline & $24-47$ & $17 \cdot 2$ & - & $5-20 \% \ddagger$ & - \\
\hline & $48-72$ & $17 \cdot 1$ & - & $10-30 \% \ddagger$ & - \\
\hline Added sugars $(g)^{*}$ & $4-6$ & $29 \cdot 5$ & $4 \cdot 3$ & No more than $25 \%$ of total energy & 24 \\
\hline & $7-9$ & $19 \cdot 9$ & $0 \cdot 1$ & & 11 \\
\hline & $10-12$ & $17 \cdot 6$ & $7 \cdot 3$ & & 10 \\
\hline & $13-23$ & $31 \cdot 4$ & $16 \cdot 5$ & & 11 \\
\hline & $24-47$ & $34 \cdot 5$ & $17 \cdot 1$ & & 11 \\
\hline & $48-72$ & $37 \cdot 3$ & $18 \cdot 0$ & & 10 \\
\hline Fibre $(\mathrm{g})^{*}$ & $4-6$ & $4 \cdot 0$ & $0 \cdot 4$ & No DRI standard & \\
\hline & $7-9$ & $8 \cdot 2$ & $0 \cdot 7$ & & \\
\hline & $10-12$ & $8 \cdot 3$ & $1 \cdot 8$ & & \\
\hline & $13-23$ & $10 \cdot 5$ & $2 \cdot 2$ & $19 \mathrm{~g}$ & 55 \\
\hline & $24-47$ & $12 \cdot 3$ & $2 \cdot 6$ & $19 \mathrm{~g}$ & 65 \\
\hline & $48-72$ & $13 \cdot 8$ & $3 \cdot 1$ & $25 \mathrm{~g}$ & 55 \\
\hline
\end{tabular}

${ }^{*}$ Recommended Dietary Intake/Adequate Intake (kcal converted to MJ). tAveraged by day, not total.

$\ddagger$ Acceptable Micronutrient Distribution Range. 
aged 10-12 months, compared with the DRI of $\sim 30 \mathrm{~g}$. There is no DRI for grams of fat for toddlers and preschool children. For children aged 4-6, 7-9, 10-12, 13-23, 24-47 and 48-72 months, menus yielded 70.3 (sD 5.9), $100 \cdot 4$ (sD 8·1), 84·1 (sD 14·0), 128 (sD 23·1), 145 (sD 23.4) and $160 \cdot 4(\mathrm{SD} 25 \cdot 4) \mathrm{g}$ carbohydrate/d and $9 \cdot 9$ (SD 0.5), $19 \cdot 8(\mathrm{sD} 0 \cdot 4), 32 \cdot 3(\mathrm{sD} 7 \cdot 0), 48 \cdot 8$ (sD $8 \cdot 8$ ), $54 \cdot 8$ (sD 9.9) and 59.9 (SD 10.0) g protein/d, respectively. Generally, the percentage of energy from fat, carbohydrate and protein was in line with DRI standards. However, the percentage of energy from fat was $38 \cdot 7 \%$ for children 48-72 months, which is slightly above the DRI recommendation of 25-35\%. According to menus, children were provided a mean daily fibre amount of $10 \cdot 5$ (sD $2 \cdot 2) \mathrm{g}$ (13-23 months), $12 \cdot 3$ (SD 2.6) g (24-47 months) and $13 \cdot 8$ (SD 3.1) g (48-72 months), which approached the DRI recommendation of 19,19 and $25 \mathrm{~g}$, respectively. Nevertheless, wholegrain foods were low for all age groups. Foods listed on the menu did not include high levels of sugar, except for sugar-sweetened milk and water. In fact, the sugar content of total daily foods was below the recommended level of $25 \%$ for all children.

\section{Discussion}

Based on dietary data from $54 \mathrm{~d}$ of menus used by 142 government-sponsored child-care centres throughout Mexico, children were provided with a high number of megajoules coming from a variety of food groups while in child care. Given that children spend part, but not all of their waking hours in child care, menus listed excessive amounts of most foods, including vegetables, fruits, meats and dairy, compared with MyPlate recommendations. Average total energy and macronutrient levels were quite high for all age groups in which DRI have been established. In particular, menus included excessive grams of protein for nearly all children. Fruit juice, sugar-sweetened beverages and full-fat milk also contributed to excessive total energy.

Several explanations may exist for the excessive amount of energy listed on the menus. Since children spend $8-10 \mathrm{~h}$ in full-day care, child-care centres may wish to provide enough energy to meet children's energy needs for an entire day, given that some children may not receive sufficient amounts foods and beverages at home. Additionally, foods listed on the menu may provide more than the recommended amounts of energy and nutrients because child-care centres may assume that children will not eat all foods and beverages served; children may elect to consume a portion of what is offered.

Despite the excess of sugar-sweetened beverages, the total amount of added sugars to foods was just over $10 \%$ of total energy intake for all age groups except infants 4-6 months of age. While this falls well below the liberal DRI recommendation of $<25 \%$, it is just over the
WHO recommendation of $<10 \%$ of daily energy and is more than double the American Heart Association recommendation of $<16 \mathrm{~g} / \mathrm{d}$ for a $5 \cdot 86 \mathrm{MJ}(1400 \mathrm{kcal}) \mathrm{diet}^{(38-40)}$. This finding was true despite pervasive amounts of sugarsweetened beverages listed on the menus. Low levels of added sugars may be due to the infrequency of processed foods; nearly all foods listed on the menu were prepared at the child-care centres from scratch, with limited reliance on pre-packaged or processed foods. Nevertheless, grains provided to children aged 48-72 months were slightly inadequate. Interestingly, fibre levels were within recommended amounts, even though wholegrain items were not. This apparent discrepancy may be due to the relatively high levels of fruits and vegetables served. Although beans are a contributor to overall fibre intake and were also listed on the menu, they did not appear frequently.

While menus suggested that the recommended average levels of dairy were served, the majority were full-fat items (for children over the age of 2 years). This finding is similar to results from Ball et al. ${ }^{(41)}$, who showed that most milk served to pre-school children in US-based child care was full-fat milk. In our sample, beverages were problematic in general, with high quantities of sugarsweetened beverages served daily. Moreover, 100\% fruit juice was provided to infants $<6$ months of age, and an average of $5 \mathrm{oz}$ of $100 \%$ fruit juice was served daily to infants aged 7-9 months. Additionally, menus listed cow's milk for infants $<12$ months of age, which goes against current recommendations to wait until children turn 1 year of age ${ }^{(42)}$.

A handful of studies have assessed the diet quality of foods and beverages served to children in child care in the USA and found that children consumed inadequate amounts of fruits and vegetables, insufficient Fe and fibre, and excessive quantities of full-fat milk ${ }^{(41,43-47)}$. Menu data from the present study suggest that the diet quality of foods served to children in child care in Mexico may be higher than in the USA, with greater amounts of fruits and vegetables served, and lower added sugars and processed foods. However, juice and cow's milk were introduced to infants too early, and children were served excessive amounts of full-fat milk, fruit juice and sugar-sweetened beverages. Beverages may be of lower nutritional quality due to the high prevalence of sugar-sweetened beverages. Because beverages contribute to children's overall energy intake, they may be an appropriate target for intervention in this setting.

The few published studies on overall dietary intake suggest that children consume inadequate fibre and excessive amounts of fat in the more urban areas of Mexico $^{(18)}$. Children also consume a high percentage of energy from beverages, including full-fat milk and fruit juice $^{(35)}$. Our findings are consistent with this previous research, in that we found menus to provide excessive total energy, insufficient whole grains and a high prevalence of full-fat milk and fruit juice. Menus were also a source of 
excessive grams of protein, and the contribution from protein made up a large percentage of overall total energy.

The present study has several limitations. First, menus were obtained from government-sponsored child-care centres and may not be representative of other types of child-care programmes in Mexico. While the study population does not represent all children in Mexico, the government-sponsored centres represent roughly onethird of all child-care centres in Mexico. Children who attend organized child care in Mexico come from smaller families with higher household incomes and are more likely to have mothers with higher educational attainment than children cared for at home ${ }^{(48)}$. Additionally, we analysed the nutrient composition of foods and beverages listed on the menu, not what was actually served to children. Previous data suggest that menus are a reasonably accurate source of information on categories of food (e.g. fruits and vegetables) actually served in child care $^{(49,50)}$. Moreover, we did not assess actual dietary intake in children, but our previous study in the USA suggests that children consume $50-100 \%$ of what they are offered in child-care centres ${ }^{(41)}$. We also compared foods, beverages and portion sizes with US-based recommendations, given the absence of specific national standards in Mexico. We did not assess foods and beverages consumed at home or away from child care, which may provide a more complete and accurate picture of dietary consumption in children $<5$ years of age.

\section{Conclusions}

The present study provides insight into the quantity and nutritional quality of foods and beverages served to young children in child-care centres in Mexico, a country with rapidly rising rates of childhood obesity. Future studies should explore dietary intake to assess what children actually consume in child care and at home throughout Mexico.

\section{Acknowledgements}

Sources of funding: This work was carried out with support from a Consejo Nacional de Ciencia y Technologia (CONACyT) grant (\#S0008-114027) and CAMBIO - Canada and Mexico Battling Childhood Obesity - which is funded by the Global Health Research Initiative (GHRI), a collaborative research funding partnership of the Canadian Institutes of Health Research, the Canadian International Development Agency, Health Canada, the International Development Research Centre and the Public Health Agency of Canada. M.W.G. was supported, in part, by a National Institutes of Health (NIH) grant (K24 HL068041). The Nutrition Obesity Research Centers (NORC) was supported by an NIH Clinical Nutrition Research Unit grant (DK56350). E.M.T. was supported, in part, by a grant from the National Center on Minority Health and Health Disparities (MD003963). Conflicts of interest: The authors report that they have no financial disclosures or competing interests. Author contributions: S.E.B.N. conceived of the study, coordinated the data analysis and drafted the manuscript. H.R.-M. and J.H. coordinated the exchange of the menus, provided guidance in the design of the study and reviewed and edited the manuscript. E.M.T. and M.W.G. provided scientific guidance on the development of the study and read and approved the manuscript. Acknowledgements: The authors would like to thank Sarah C. Ball for her assistance compiling results for this manuscript, as well as the NORC at the University of North Carolina-Chapel Hill for their assistance with the nutrient analysis.

\section{References}

1. Romieu I, Hernandez-Avila M, Rivera J et al. (1997) Dietary studies in countries experiencing a health transition: Mexico and Central America. Am J Clin Nutr 65, 4 Suppl., 1159S-1165S.

2. Rivera JA, Barquera S, González-Cossío T et al. (2004) Nutrition transition in Mexico and in other Latin American countries. Nutr Rev 62, 7 Pt 2, S149-S157.

3. Fernald LC \& Neufeld LM (2006) Overweight with concurrent stunting in very young children from rural Mexico: prevalence and associated factors. Eur J Clin Nutr 61, 623-632.

4. Rivera JA, Monterrubio EA, Gonzalez-Cossio T et al. (2003) Nutritional status of indigenous children younger than five years of age in Mexico: results of a national probabilistic survey. Salud Publica Mex 45, Suppl. 4, S466-S476.

5. Rivera JA \& Sepulveda Amor J (2003) Conclusions from the Mexican National Nutrition Survey 1999: translating results into nutrition policy. Salud Publica Mex 45, Suppl. 4, S565-S575.

6. Jaime S, Flavia B, Roberto T et al. (2006) Improvement of child survival in Mexico: the diagonal approach. Lancet 368, 2017-2027.

7. Rivera JA, Barquera S, Campirano F et al. (2002) Epidemiological and nutritional transition in Mexico: rapid increase of non-communicable chronic diseases and obesity. Public Health Nutr 5, 113-122.

8. Bonvecchio A, Safdie M, Monterrubio EA et al. (2009) Overweight and obesity trends in Mexican children 2 to 18 years of age from 1988 to 2006. Salud Publica Mex 51, Suppl. 4, S586-S594.

9. del Rio-Navarro BE, Velazquez-Monroy O, Sanchez-Castillo CP et al. (2004) The high prevalence of overweight and obesity in Mexican children. Obes Res 12, 215-223.

10. Fernald LC, Gutierrez JP, Neufeld LM et al. (2004) High prevalence of obesity among the poor in Mexico. JAMA 291, 2544-2545.

11. Filozof C, Gonzalez C, Sereday M et al. (2001) Obesity prevalence and trends in Latin-American countries. Obes Rev 2, 99-106.

12. Sanchez-Castillo CP, Lara JJ, Villa AR et al. (2001) Unusually high prevalence rates of obesity in four Mexican rural communities. Eur J Clin Nutr 55, 833-840.

13. Bacardi-Gascón M, Jiménez-Cruz A, Jones E et al. (2009) Trends of overweight and obesity among children in Tijuana, Mexico. Ecol Food Nutr 48, 226-236.

14. Villa-Caballero L, Caballero-Solano V, Chavarría-Gamboa M et al. (2006) Obesity and socioeconomic status in children of Tijuana. Am J Prev Med 30, 197-203. 
15. Freedman DS, Serdula MK, Srinivasan SR et al. (1999) Relation of circumferences and skinfold thicknesses to lipid and insulin concentrations in children and adolescents: the Bogalusa Heart Study. Am J Clin Nutr 69, 308-317.

16. Pinhas-Hamiel O, Dolan LM, Daniels SR et al. (1996) Increased incidence of non-insulin-dependent diabetes mellitus among adolescents. J Pediatr 128, 608-615.

17. Morrison JA, Sprecher DL, Barton BA et al. (1999) Overweight, fat patterning, and cardiovascular disease risk factors in black and white girls: The National Heart, Lung, and Blood Institute Growth and Health Study. J Pediatr 135, 458-464.

18. Colín-Ramírez E, Castillo-Martínez L, Orea-Tejeda A et al. (2009) Waist circumference and fat intake are associated with high blood pressure in Mexican children aged 8 to 10 years. J Am Diet Assoc 109, 996-1003.

19. Leung TF, Li CY, Lam CW et al. (2004) The relation between obesity and asthmatic airway inflammation. Pediatr Allergy Immunol 15, 344-350.

20. Perez-Padilla R, Rojas R, Torres V et al. (2006) Obesity among children residing in Mexico City and its impact on lung function: a comparison with Mexican-Americans. Arch Med Res 37, 165-171.

21. Taveras EM, Rifas-Shiman SL, Camargo CA et al. (2008) Higher adiposity in infancy associated with recurrent wheeze in a prospective cohort of children. J Allergy Clin Immunol 121, 1161-1166. e1163.

22. Vignolo M, Naselli A, Di Battista E et al. (1988) Growth and development in simple obesity. Eur J Pediatr 147, 242-244.

23. Lee JM, Appugliese D, Kaciroti N et al. (2007) Weight status in young girls and the onset of puberty. Pediatrics 119, e624-630.

24. French SA, Story M \& Perry CL (1995) Self-esteem and obesity in children and adolescents: a literature review. Obes Res 3, 479-490.

25. Puhl RM \& Latner JD (2007) Stigma, obesity, and the health of the nation's children. Psychol Bull 133, 557-580.

26. Puhl RM, Moss-Racusin CA \& Schwartz MB (2007) Internalization of weight bias: implications for binge eating and emotional well-being. Obesity (Silver Spring) 15, $19-23$.

27. Malik VS, Schulze MB \& Hu FB (2006) Intake of sugarsweetened beverages and weight gain: a systematic review. Am J Clin Nutr 84, 274-288.

28. Dennison BA, Rockwell HL \& Baker SL (1997) Excess fruit juice consumption by preschool-aged children is associated with short stature and obesity. Pediatrics 99, 15-22.

29. Faith MS, Dennison BA, Edmunds LS et al. (2006) Fruit juice intake predicts increased adiposity gain in children from low-income families: weight status-by-environment interaction. Pediatrics 118, 2066-2075.

30. Taveras EM, Berkey CS, Rifas-Shiman SL et al. (2005) Association of consumption of fried food away from home with body mass index and diet quality in older children and adolescents. Pediatrics 116, e518-524.

31. Bowman SA, Gortmaker SL, Ebbeling CB et al. (2004) Effects of fast-food consumption on energy intake and diet quality among children in a national household survey. Pediatrics 113, 112-118.

32. Brownell KD (2004) Fast food and obesity in children. Pediatrics 113, 132.
33. Lozada M, Sánchez-Castillo CP, Cabrera GA et al. (2008) School food in Mexican children. Public Health Nutr 11, 924-933.

34. Mundo-Rosas V, Rodríguez-Ramírez S \& Shamah-Levy T (2009) Energy and nutrient intake in Mexican children 1 to 4 years old. Results from the Mexican National Health and Nutrition Survey 2006. Salud Publica Mex 51, Suppl. 4, S530-S539

35. Barquera S CF, Bonvecchio A, Hernandez-Barrera L et al. (2010) Caloric beverage consumption patterns in Mexican children. Nutr J 9, 1475-2891.

36. US Department of Agriculture (2009) Daily Food Plan for Preschoolers. http://www.choosemyplate.gov/preschoolers/ daily-food-plans.html (accessed March 2012).

37. Office of Dietary Supplements, National Institutes of Health (2009) Nutrient Recommendations: Dietary Reference Intakes (DRI) http://ods.od.nih.gov/health_information/ Dietary_Reference_Intakes.aspx (accessed September 2012).

38. Nishida C, Uauy R, Kumanyika S et al. (2004) The joint WHO/FAO expert consultation on diet, nutrition and the prevention of chronic diseases: process, product and policy implications. Public Health Nutr 7, 245-250.

39. National Research Council (2005) Dietary Reference Intakes for Energy, Carbohydrate, Fiber, Fat, Fatty Acids, Cholesterol, Protein, and Amino Acids (Macronutrients). Washington, DC: The National Academies Press.

40. Johnson RK, Appel LJ, Brands M et al. (2009) Dietary sugars intake and cardiovascular health: a scientific statement from the American Heart Association. Circulation 120, 1011-1020.

41. Ball SC, Benjamin SE \& Ward DS (2008) Dietary intakes in North Carolina child-care centers: are children meeting current recommendations? J Am Diet Assoc 108, 718-721.

42. American Academy of Pediatrics (2003) Prevention of pediatric overweight and obesity. Pediatrics 112, 424-430.

43. Briley ME, Buller AC, Roberts-Gray CR et al. (1989) What is on the menu at the child care center? J Am Diet Assoc 89, 771-774.

44. Padget A \& Briley ME (2005) Dietary intakes at child-care centers in central Texas fail to meet Food Guide Pyramid recommendations. J Am Diet Assoc 105, 790-793.

45. Oakley CB, Bomba AK, Knight KB et al. (1995) Evaluation of menus planned in Mississippi child-care centers participating in the Child and Adult Care Food Program. J Am Diet Assoc 957, 765-768.

46. Erinosho T, Dixon LB, Young C et al. (2011) Nutrition practices and children's dietary intakes at 40 child-care centers in New York city. J Am Diet Assoc 111, 1391-1397.

47. Sigman-Grant M, Christiansen E, Branen L et al. (2008) About feeding children: mealtimes in child-care centers in four western states. J Am Diet Assoc 108, 340-346.

48. Flores Hernández S, Reyes Morales H, Pérez Cuevas R et al. (1999) The day care center as a risk factor for acute respiratory infections. Arch Med Res 30, 216-223.

49. Benjamin Neelon SE, Copeland KA, Ball SC et al. (2010) Comparison of menus to actual foods and beverages served in North Carolina child-care centers. J Am Diet Assoc 110, 1890-1895.

50. Fleischhacker S, Cason KL \& Achterberg C (2006) 'You had peas today?': a pilot study comparing a Head Start child-care center's menu with the actual food served. J Am Diet Assoc 106, 277-280. 